\title{
Mesoscopic circuits with charge discreteness: quantum transmission lines
}

\author{
J. C. Flores \\ Universidad de Tarapacá, Departamento de Física, Casilla 7-D, \\ Arica, Chile
}

We propose a quantum Hamiltonian for a transmission line with charge discreteness. The periodic line is composed of an inductance and a capacitance per cell. In every cell the charge operator satisfies a nonlinear equation of motion because of the discreteness of the charge. In the basis of one-energy per site, the spectrum can be calculated explicitly. We consider briefly the incorporation of electrical resistance in the line.

PACS Numbers: 73.23.-b ; 73.21.-b ; 73.63.-b ; 07.50.Ek

Will be published in Phys.Rev.B 


\section{Introduction: LC quantum circuits with charge discreteness}

Nowadays technological miniaturization of circuits is increasing and their mesoscopic aspects become more relevant. Recently, a theory for mesoscopic circuits was proposed by Li and Chen [1] where charge discreteness was considered explicitly. This is very related to miniaturization since the number of charges in these systems is expected to be more and more reduced. In the $L C$ circuit studied in reference [1], the Hamiltonian operator was given by

$$
\widehat{H}=\widehat{T}+\widehat{V},
$$

where $V(\widehat{Q})=\widehat{Q}^{2} / 2 C$, with $\widehat{Q}$ the charge operator, is the electrical energy in the capacitance $C$ and the magnetic energy operator term $\widehat{T}$, related to the inductance $L$, was given by

$$
\widehat{T}=\frac{2 \hbar^{2}}{L q_{e}^{2}}\left(\sin ^{2}\left(\frac{L q_{e}}{2 \hbar} k\right)\right),
$$

in $k$-representation (or pseudo-current representation, $0<k<2 \pi$ ). The above operator has some resemblance with a mechanical kinetic operator in the limit of small $k$. In (2) the constant $q_{e}$ represents the elementary charge in the electric system. In this representation, the charge operator $\widehat{Q}$ is given by

$$
\widehat{Q}=\frac{i \hbar}{L} \frac{\partial}{\partial k}
$$

and the corresponding eigenfunctions are $e^{i n L q_{e} k / \hbar}$ with discrete eigenvalues $n q_{e}$ where $n$ is an integer. Since the current operator $\widehat{I}$ is formally obtained from $\widehat{I}=\left(\frac{1}{i \hbar}\right)[\widehat{H}, \widehat{Q}]$ then, in the $k$-representation, it becomes

$$
\widehat{I}=\frac{\hbar}{L q_{e}} \sin \left(\frac{L q_{e}}{\hbar} k\right) .
$$

In the limit $q_{e} \rightarrow 0$ all these operators become the usual ones associated with a quantum $L C$ circuit with continuous charge [3]. Moreover, the current operator (4) is bounded with extrema values $\pm \frac{\hbar}{L q_{e}}$. After reference [1], the 
above Hamiltonian describes phenomena like persistent current, Coulomb blockage and others.

As pointed-out in [2], the above set of operators defines an algebra with some similitude to this related to space-time discreteness $[4,5,6]$. In fact, the charge-current commutator $[\widehat{Q}, \widehat{I}]$, usually proportional to the identity, becomes modified with a kinetic term which is zero when $q_{e}=0$. So, space and charge discreteness could be described with the same mathematical tools.

On the other hand, dissipation is a subject very related to electric circuits. This phenomenon defines a decoherence-time related to mesoscopic aspect. Dissipation can be considered in many forms, the usual one is to connect the system to a bath with many degree of freedom, and with some assumptions respect to the decaying rate [7]. Phenomenological $R L C$ circuits can be also considered using Caldirola-Kanai theory $[8,9,10]$ in a direct way [11].

In this paper we are interested in a quantum transmission line. Such transmission lines are usually used in mesoscopic physics. For instance, in reference [12], a quantum transmission line with continuous charge was considered and connected to a metal ring. It was quantized and used as environment to study zero-point fluctuations influence on a metal ring. Particularly, we shall consider a quantum transmission line with charge discreteness. For this purpose we shall use the ideas discussed above. In this way, we consider a periodic transmission line composed of cells. Every cell has an inductance $L$ and a capacitance $C$.

In section II, we consider briefly the classical transmission line which will be quantized by standard procedure. In section III, we present the quantized Hamiltonian which contains explicitly charge discreteness. Also in that section, we write the motion equation of the charge in the line using Heinsenberg equation of motion. In section IV, in the one-energy per site approximation, we find the spectral properties of the systems. In section $\mathrm{V}$, we study briefly the incorporation of electrical resistance in the quantum transmission line with charge discreteness. In the last section, conclusions are touched. 


\section{Classical transmission line}

In this section we shall consider a periodic transmission line composed in every cell of an inductance $L$ and a capacitance $C$. Classically the evolution equation for the continuous charge $Q_{l}$, at cell $l(l \in Z)$ in the transmission line, is given by the expression

$$
L \frac{d^{2}}{d t^{2}} Q_{l}=\frac{1}{C}\left(Q_{l+1}+Q_{l-1}-2 Q_{l}\right),
$$

which can be obtained from the classical Hamiltonian given by

$$
H_{\text {clas }}=\sum_{l} \frac{\phi_{l}^{2}}{2 L}+\frac{1}{2 C}\left(Q_{l+1}-Q_{l}\right)^{2},
$$

where the variable $\phi_{l}$ corresponds to the magnetic flux in the inductance at position $l$ and it is proportional to the classical current in the cell. It is explicitly given by

$$
\phi_{l}=L \frac{d Q_{l}}{d t} .
$$

The integer $l$ in (5) represents the index of the cell at position $l$. The first term depending on the current in (6) is the equivalent to the kinetic energy of a mechanical system and is related to the stored magnetic energy in the inductance. In the quadratic potential, the crossed term $\left(Q_{l+1} Q_{l} / C\right)$ represents the interaction term between cells. In fact, for continuous variables, the above Hamiltonian is equivalent to this one of mechanical vibrations and could be quantized directly. By canonical transformation, the classical Hamiltonian (6) could be transformed to normal modes and its spectral fre-

quencies are well-known. For us, the important fact is that the crossed term in (6) defines the interaction between two consecutive sites or cells. This interaction term will be preserved in the quantization process. 


\section{A Hamiltonian for quantum transmission lines with charge discreteness}

Due to charge discreteness in the quantum case, the structural changes are only expected to occur in the kinetic part of (6) where the usual quadratic term is transformed in a trigonometric function (section I). Then, the quantum transmission line with charge discreteness can be quantized directly. So, from the classical Hamiltonian (6), and equations (2) and (3), the quantum Hamiltonian for the transmission line with charge discreteness is

$$
\widehat{H}=\sum_{l}\left\{\frac{2 \hbar^{2}}{L q_{e}^{2}} \sin ^{2}\left(\frac{L q_{e}}{2 \hbar} k_{l}\right)-\frac{\hbar^{2}}{2 L^{2} C}\left(\frac{\partial}{\partial k_{l+1}}-\frac{\partial}{\partial k_{l}}\right)^{2}\right\}
$$

where the quantity $k_{l}$ corresponds to the pseudo-current in the cell at position $l$ and varies between $0<k_{l}<2 \pi$. As pointed before, the kinetic part is not quadratic in $k_{l}$, which is very related to the charge discreteness assumptions $\left(q_{e} \neq 0\right)$. The study of the Hamiltonian (8) is the purpose of this work. Remark that in the limit $q_{e} \rightarrow 0$ the kinetic part becomes proportional to $k_{l}^{2}$ with formal similitude to the case of mechanical vibrations (phonons). This corresponds to the continuous charge case and the name circuitons for these propagating modes is appropriate. Nevertheless, circuitons are the limit case with zero charge discreteness $q_{e} \rightarrow 0$. In a general context, since the above Hamiltonian describes the quantization of the line and the quantization of the charge we shall call cirquitons the normal modes (propagating modes) of the above Hamiltonian. This appellation seems appropriate because it remembers: (i) the quantization process and (ii) the discreteness of the charge $\left(q_{e}\right)$. The existence of these propagating modes is ensured since the Hamiltonian (8) is invariant under (discrete) spatial traslation.

The motion equation related to the quantum transmission line can be found with the usual quantum mechanical evolution rules (Heisenberg equations). The pseudo-current $k_{l}$ has associated the canonical conjugate operator

of the charge, namely, the operator $\widehat{K}_{l}$ with eigenvalues $k_{l}$. It satisfies the canonical commutation relation : 


$$
L\left[\widehat{Q}_{l}, \widehat{K}_{l}\right]=i \hbar .
$$

As function of this operator, the Hamiltonian of the transmission line becomes

$$
\widehat{H}=\sum_{l}\left\{\frac{2 \hbar^{2}}{L q_{e}^{2}} \sin ^{2}\left(\frac{L q_{e}}{2 \hbar} \widehat{K}_{l}\right)+\frac{1}{2 C}\left(\widehat{Q}_{l+1}-\widehat{Q}_{l}\right)^{2}\right\},
$$

and the evolution equation for the charge operator in the Heinsenberg representation $\frac{d}{d t} \widehat{Q}_{l}=\frac{i}{\hbar}\left[\widehat{H}, \widehat{Q}_{l}\right]$ can be computed explicitly:

$$
\frac{d}{d t} \widehat{Q}_{l}=\frac{\hbar}{q_{e} L} \sin \left(\frac{L q_{e}}{\hbar} \widehat{K}_{l}\right) .
$$

Which is similar to the nonlinear expression (4) for every cell. In fact, (11) defines the current operator and it is bounded like to the case mentioned in the section I. The motion equation for the pseudo-current operator $\frac{d}{d t} \widehat{K}_{l}=$ $\frac{i}{\hbar}\left[\widehat{H}, \widehat{K}_{l}\right]$ is

$$
\frac{d}{d t} \widehat{K}_{l}=\frac{1}{C L}\left(\widehat{Q}_{l+1}+\widehat{Q}_{l-1}-2 \widehat{Q}_{l}\right) .
$$

In equations (11) and (12), we notice that the formal limit $q_{e} \rightarrow 0$ gives the usual linear motion equation for transmission lines with similitude to the classical one (5). Nevertheless, in the general case, the evolution equation is nonlinear.

\section{One-energy per site: excitations on the transmission line}

The study of the spectral properties of the Hamiltonian (8), or (10), is difficult because of the nonlinear term associated with the magnetic energy. Without charge discreteness $\left(q_{e} \rightarrow 0\right)$, this operator is quadratic and the usual normal modes technique could be used [13]. In this section we solve the spectral properties on the vector-basis of the system without interaction. 
We shall find a particular spectral solution in the basis of one-energy per site or cell.

The Hamiltonian (10) can be written as

$$
\widehat{H}=\sum_{l} \widehat{H}_{l}-\frac{1}{C} \widehat{Q}_{l+1} \widehat{Q}_{l},
$$

where the site-Hamiltonian $\widehat{H}_{l}$ corresponds to this one of a $L C$ circuit (1) with capacitance $C / 2$. Since the total Hilbert space, where the Hamiltonian (13) acts, is the direct product of the spaces associated to every site $l$, then we consider the sub-basis $\{|l\rangle, l \in Z\}$ where every element $|l\rangle$ is an eigenstate of the Hamiltonian $\widehat{H}_{l}$ with energy $V$. Namely,

$$
\widehat{H}_{l}|l\rangle=V|l\rangle \text {. }
$$

Since $\langle n \mid l\rangle=\delta_{l, n}$ and the operator $\widehat{Q}_{l}$ acts only in the site $l$, the complete Hamiltonian $\widehat{H}$ is Hermitian and becomes tri-diagonal in this basis. Explicitly, it is given by the matrix elements:

$$
\langle l|\widehat{H}| l\rangle=V ; \text { and }\langle l|\widehat{H}| l+1\rangle=-\alpha \frac{q_{e}^{2}}{C},
$$

where $\alpha$ is a dimensionless constant which we keep as one $(\alpha=1)$. Formally, this can be carried-out by an adequate normalization. Noticed that the offdiagonal terms in (15) are related to the interacting term in (13), namely, $\frac{1}{C} \widehat{Q}_{l+1} \widehat{Q}_{l}$ and then it was expected to be proportional to $q_{e}^{2}$.

The $L C$ energy stored in a cell is now spread into a band due to charge interaction with the two neighboring cells. The spectrum of the tri-diagonal Hamiltonian is well-known and corresponds to the so-called tight-binding approximations in Solid State Physics. In fact, assuming a general state of the form $|\psi\rangle=\sum \psi_{l}|l\rangle$, the Schrödinger equation related to the tri-diagonal Hamiltonian (15) becomes:

$$
E \psi_{l}=V \psi_{l}-\frac{q_{e}^{2}}{C}\left(\psi_{l+1}+\psi_{l-1}\right)
$$

with $E$ the energy. The eigenstates are of the form $\psi_{l}=e^{i \theta l}$, where the phase $\theta$ is a real number $(0<\theta<2 \pi)$, and the spectrum becomes 


$$
E=V-\frac{2 q_{e}^{2}}{C} \cos \theta
$$

where we have considered an infinite transmission line (i.e. $\theta$ is a continuous variable). The above expression defines the density of states and the thermodynamics properties of these lines could be calculated.

The spectrum (17) defines an ensemble of quantum excitations on the transmission line, nevertheless, they are not the more general because they are only defined in the sub-space spanned by $\{|l\rangle, l \in Z\}$ in the complete Hilbert space. For instance, others kind of excitations could be found if we consider two energies by site, or different energies by site.

\section{Transmission lines with resistance}

The Hamiltonian (10) describes a quantum transmission line with charge discreteness, but it does not consider dissipation. On the other hand, electrical resistance (Ohm law) is an intrinsic phenomenon in electrical conduction. After Bateman's work [14], classical linear dissipation could be studied in a Lagrangian way by consider a time-decaying exponential factor multiplying the Langrangian function. From this, the classical Hamiltonian could be written in the standard way. So, quantization becomes attainable from the usual correspondence between position-momentum and its associated operators $[8,9,10]$. In our case, a similar procedure could be implemented and we obtain the time depending Hamiltonian:

$$
\widehat{H}(t)=\sum_{l}\left\{e^{-\frac{R}{L} t} \frac{2 \hbar^{2}}{L q_{e}^{2}} \sin ^{2}\left(\frac{L q_{e}}{2 \hbar} \widehat{K}_{l}\right)+e^{\frac{R}{L} t} \frac{1}{2 C}\left(\widehat{Q}_{l+1}-\widehat{Q}_{l}\right)^{2}\right\},
$$

where the constant $R$ represents the resistance. With the above Hamiltonian, and the Heinsenberg motion equations, we obtain for the charge operator

$$
\frac{d}{d t} \widehat{Q}_{l}=\frac{\hbar}{q_{e} L} e^{-\frac{R}{L} t} \sin \left(\frac{L q_{e}}{\hbar} \widehat{K}_{l}\right)
$$


and for the pseudo-current operator

$$
\frac{d}{d t} \widehat{K}_{l}=\frac{1}{C L} e^{\frac{R}{L} t}\left(\widehat{Q}_{l+1}+\widehat{Q}_{l-1}-2 \widehat{Q}_{l}\right) .
$$

From (19) and (20), the equation for the variation of the charge becomes

$$
L \frac{d^{2}}{d t^{2}} \widehat{Q}_{l}=-R \frac{d}{d t} \widehat{Q}_{l}+\frac{1}{C}\left(\cos \left(\frac{L q_{e}}{\hbar} \widehat{K}_{l}\right)\right)\left(\widehat{Q}_{l+1}+\widehat{Q}_{l-1}-2 \widehat{Q}_{l}\right)
$$

which in the formal limit $q_{e} \rightarrow 0$ becomes the usual one incorporating a resistance $R$ in every cell of the transmission line. So, the time depending Hamiltonian (18) describes the dynamics of a quantum transmission line with charge discreteness and resistance.

\section{Conclusions and discussions}

We have proposed a quantum Hamiltonian for a transmission line composed of a periodic array of inductances and capacitances with charge discreteness $((8)$ or $(10))$. To construct this Hamiltonian we have used the charge discreteness procedure proposed in reference [1] for a $L C$ circuit. In our case, the corresponding Hilbert space is given by the tensorial product of this one of every cell, corresponding to a $L C$ circuit. In the particular basis of one-energy per site, or cell, we have found the spectrum of the line (17).

Note that charge discreteness produces nonlinear terms in the equation of motion in the transmission line $(11,12)$. The incorporation of electrical resistance was considered by using the Caldirola-Kanai theory (section V).

As a final remark we note that disorder systems are usually studied in Solid State Physics [15] and it is well-known that localization of states could exist. In our case, we believe that disorder can be incorporated in the line by consider for instance every inductance as a random quantity. In this case, it seems interesting to study the role of disorder and the nonlinearity due to charge discreteness. On the other hand, it is known that decoherence effects 
break localization $[16,17]$ then the role of environment and resistance on the transmission line must also break localization.

Acknowledgments: Discussion about the role of charge discreteness in mesoscopic systems were carried-out with P. Orellana (Universidad Católica del Norte). Useful discussion were carried-out at the II International Workshop on Disordered Systems (Arica, UTA 2000). This work was supported by FONDECYT (Project 1990443).

\section{References}

[1] You-Quan Li and Bin Chen, Phys.Rev.B 53, 4027 (1996).

[2] You-Quan Li, Spin-Statistical Connection and Commutation Relations. AIP Conference Proceeding Vol. 545, pp 85 (2000). You-Quan Li, Commutation relation in mesoscopic electric circuits, cond-mat/0009352 (unpublished).

[3] W. H. Louisell, Quantum Statistical Properties of Radiation, John Wiley, New York (1973).

[4] H. S. Snyder, Phys.Rev. 71, 38 (1947).

[5] I. Saavedra and C. Utreras, Phys.Lett.B 98, 74 (1981).

[6] S. Montecinos, I. Saavedra and O. Kunstmann, Phys.Lett.A 109, 131 (1985).

[7] C. Gardiner, Handbook of Stochastic Methods, Springer-Verlag, Berlin (1990).

[8] P. Caldirola, Nuovo Cimento 18, 393 (1941).

[9] E. Kanai, Prog.Theor.Phys. 3, 440 (1948). 
[10] H-J Wagner, Z.phys. B 95, 261 (1994).

[11] J. C. Flores, cond-mat/9908012 (unpublished).

[12] P. Cedraschi, V. V. Ponomarenko and M. Büttiker, Phys.Rev.Lett. 84, 346 (2000).

[13] D. Pines, Elementary Excitations In Solid, Perseus Books (1999).

[14] H. Bateman, Phys.Rev. 38, 815 (1931).

[15] P. W. Anderson, Phys.Rev. 109, 1492 (1958).

[16] J. C. Flores, Phys.Rev.B, 60, 30 (1999).

[17] S. A. Gurvitz, Phys.Rev.Lett.85, 812 (2000). 